Recepción: 02 / 05 / 2018

Aceptación: 05 / 06 / 2018

Publicación: 02 / 07 / 2018

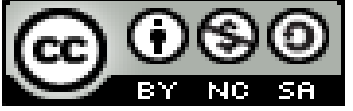

Ciencias de la salud

Artículo de Investigación

\title{
La violencia obstétrica como elemento deshumanizador del parto
}

\author{
Obstetric violence as a dehumanizing element of childbirth
}

Violência obstétrica como elemento desumanizador do parto

\author{
Delia N. Crespo-Antepara ${ }^{\mathrm{I}}$ \\ deliacrespo57@hotmail.com \\ Bryan M. Sánchez-Zambrano II \\ bmsanchezzamb@hotmail.com \\ Doménica P. Domínguez-Ontano ${ }^{\mathrm{III}}$ \\ domenicadixi@hotmail.com
}

Correspondencia: deliacrespo57@hotmail.com

\begin{abstract}
I Magister en Gerencia Clínica en Salud Sexual y Reproductiva, Diplomado en Docencia Superior, Obstetriz, Docente en la Universidad de Guayaquil, Guayaquil, Ecuador.

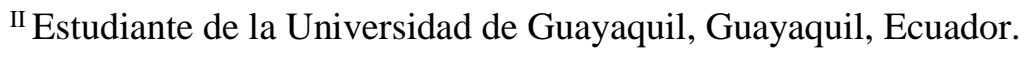

III Estudiante de la Universidad de Guayaquil, Guayaquil, Ecuador.
\end{abstract}




\section{Resumen}

La violencia obstétrica, son todas las acciones y conductas que deshumanizan a las mujeres durante el proceso del parto; bajo esta premisa, este artículo evaluó la existencia de violencia obstétrica en la atención recibida en los Hospitales Matilde Hidalgo de Procel, General Guasmo Sur y Mariana de Jesús de Guayaquil. La metodología siguió una tipología descriptiva de corte transversal. Los datos se obtuvieron posterior a la aplicación de un instrumento tipo cuestionario a las usuarias del área de la consulta externa, sala de post parto y hospitalización, utilizando como técnica la encuesta. Entre los resultados obtenidos, se pudo constatar que el 59.33\% (89) de las usuarias recibieron comentarios inapropiados, $77.33 \%$ (116) fueron llamadas con sobrenombres, y $36 \%$ (54) refirieron agresión verbal; al 72\% (108) de las usuarias no se les permitió optar por una posición de preferencia, al 72.67\% (109) se les negó el acompañamiento de una persona de confianza, y el $65.33 \%$ (98) no tuvo la oportunidad del apego precoz. En lo referente a procedimientos realizados para acelerar el parto, estos fueron $100 \%$ de estos fueron realizados sin consentimiento informado, 48\% (72) de las usuarias se les practicó episiotomía, 32.67\% (49) recibieron conducción y $11.33 \%$ (17) se les realizó rotura artificial de membranas. Entre las conclusiones, se pudo determinar que sí existe violencia obstétrica, y en algunos casos la percepción por parte de las usuarias es en alto grado.

Palabras clave: violencia obstétrica; parto; elemento deshumanizador; parturienta.

\section{Abstract}

Obstetric violence are all the actions and behaviors that dehumanize women during the process of delivery, based on this premise, this article evaluated the existence of violence obstetric care received in the hospitals Matilde Hidalgo de Procel, General Guasmo sur and Mariana de Jesús of Guayaquil. The methodology followed a cross-sectional descriptive typology. Data obtained after the application of an instrument type questionnaire to users on the area of the external consultation, post-partum and hospitalization, as a technique using the survey. Among the obtained results, it was found that the 59.33\% (89) users received inappropriate comments, $77.33 \%$ (116) were called with nicknames and $36 \%$ (54) was referred to verbal aggression; the $72 \%$ (108) users were not allowed to opt out of a position in preference to the $72.67 \%$ (109) he refused them accompanied by a person of trust, and the $65.33 \%$ (98) did not have the opportunity of early attachment. In relation to procedures to speed up the delivery, these were $100 \%$ of these 
were performed without informed consent, $48 \%$ users (72) was practiced episiotomy, 32.67\%

(49) were driving and $11.33 \%$ (17) underwent break arti mination of membranes. Among the conclusions, that obstetric violence there and in some cases the perception by users is in high grade could be determined.

Keywords: obstetric violence; childbirth; deshumanizador element; parturient.

\section{Resumo}

Violência obstétrica são todas as ações e comportamentos que desumanizam as mulheres durante o processo de nascimento; Sob esta premissa, este artigo avaliou a existência de cuidados obstétricos de violência nos hospitais Matilde Hidalgo, General Guasmo Sul e Mariana de Jesus de Guayaquil. A metodologia seguiu uma tipologia descritiva de seção transversal. Os dados foram obtidos após a aplicação de um questionário para a área de utilizador do instrumento de consulta externa, quarto pós-parto e tipo de hospital, utilizando a técnica de levantamento. Entre os resultados, verificou-se que 59,33\% (89) de utilizadores recebido comentários inadequados, $77,33 \%$ (116) foram chamados com nicks, e 36\% (54) relataram abuso verbal; 72\% (108) os utilizadores não poderão optar por uma posição preferida, a 72,67\% (109) foi negado o acompanhamento de uma pessoa de confiança e 65,33\% (98) não teve nenhuma possibilidade de fixação precoces procedimentos relativos realizada para acelerar o parto, estas foram de $100 \%$ destes foram realizados sem o consentimento informado, 48\% (72) dos utilizadores foram submetidos a episiotomia, 32,67\% (49) recebido de condução e de 11,33\% (17) ele realizou a ruptura artificial de membranas. Entre os resultados, foi determinado que existe a violência obstétrica, e em alguns casos a percepção dos usuários é alta.

Palavras chave: violência obstétrica; parto elemento desumanizador; parturiente.

\section{Introducción}

Aun cuando estamos conscientes que el parto es un proceso natural y fisiológico, por los cuales atraviesan todas las mujeres en edad reproductiva, es importante destacar que las mismas tienen derecho a recibir atención digna sin discriminación de ninguna naturaleza, criterios que coinciden con los declarados por la Organización Mundial de la Salud (2014), que bajo una declaración "El nacimiento no es una enfermedad”(p.11), pretende preservar el carácter 
natural del proceso del parto y reducir las tasas de cesáreas a menos del 15\%,así como evitar el uso inadecuado de la tecnología obstétrica.

Destacadas investigaciones sobre experiencias de mujeres en el momento del parto, plantean una situación muy alarmante, en la que es común escuchar relatos de mujeres acerca de trato irrespetuoso, ofensivo o negligente durante el parto, en la que intervienen múltiples variables con una perspectiva antropológica, social, cultural, y psicológica. En este sentido, señalan Teran, Castellanos, Gonzalez \& Ramos (2013), que la violencia obstétrica son todas las acciones y conductas que deshumanizan y minimizan a las mujeres durante todo su proceso de embarazo, parto y la etapa posterior y son cometidos por el sistema de salud tanto público como privado.

Este tipo de violencia se manifiesta a través de malos tratos, humillaciones, no proporcionando información sobre los tratamientos y tiene como consecuencia la pérdida de libertad, autonomía y capacidad de decidir libremente sobre sus cuerpos y sexualidad. Posición que hace importante el estudio sobre la violencia obstétrica, siendo esta la principal causa de vulnerabilidad de los derechos sexuales y reproductivos e incluso constitucionales, atentando contra la integridad psicológica, social y física de las usuarias.

Es de notar, que muchas de estas experiencias pueden resultar traumáticas y van más allá de las mujeres embarazas, ya que los malos tratos también pueden ser dirigidos a sus familiares. De allí, que una conducta inadecuada del profesional durante la atención obstétrica al momento del parto vulneran e irrespetan los derechos de la mujer, haciendo necesario establecer la existencia o no de violencia obstétrica, ya que no solo sirve para interpretar hallazgos sino para entender la significación de las diferentes razones de violencia obstétrica, es decir todo aquello que la sociedad desconoce y que puede producir repercusiones en torno a la calidad de vida de las parturientas extensivas a sus familiares, lo que hizo necesario indagar en qué medida no se cumple con este precepto en los hospitales de la ciudad de Guayaquil, a partir de la apreciación de las usuarias del servicio de obstetricia.

\section{Desarrollo}

De acuerdo a la Ley Orgánica para la Atención del Parto Humanizado (2012), la Violencia Obstétrica son todas las acciones y conductas que deshumanizan y minimizan a las mujeres durante todo su proceso de embarazo, parto y la etapa posterior y son cometidos por el sistema de 
salud tanto público como privado; es considerada como una violación de los Derechos Humanos, incluyendo los derechos de género a pesar de que en algunos países de Latinoamérica está contemplada como delito dentro de la constitución. Sin embargo, aún se observa que hay hospitales y profesionales que continúan vulnerando estos derechos.

Es por ello, que reviste un carácter urgente promover la reflexión y sensibilización de los agentes de salud sobre este tema particular para lograr modificar aquellas prácticas en salud violatorias de los derechos de las mujeres, en la que se debe garantizar el derecho de las mujeres a contar con toda la información relacionada con su tratamiento, así como el derecho a recibir por parte del personal de salud el mejor trato posible, teniendo en cuenta las limitaciones de los servicios de salud sexual y reproductiva, garantizando la igualdad del acceso y la calidad de la atención independientemente de la procedencia socioeconómica o cultural de la mujer.

Siendo así, los Estados deben garantizar estos derechos a través de políticas y programas para mejorar la atención en salud de las mujeres, incorporando la perspectiva de género, los derechos humanos y la atención culturalmente adecuada antes, durante y después del parto.

La violencia obstétrica incluye:

$\checkmark$ Comentarios hirientes como "si te gustó hacerlo, ahora aguántate", "así gritaste cuando lo estabas haciendo", "golpes en las piernas".

$\checkmark$ Impedir a las usuarias expresarse durante el proceso del trabajo de parto o parto: "ya no llores, no es para tanto".

$\checkmark$ Comentarios pocos apropiados con la sexualidad de las mujeres.

$\checkmark$ Escasa información o falta de la misma.

$\checkmark$ Llevar a cabo procedimientos médicos de forma rutinaria, sin consentimiento o explicarle por qué son tan necesarios realizarlos, incluso cuando no son necesarios para facilitar el proceso o apresurar los tiempos ejemplo; (abuso de oxitócicos para apresurar el parto).

$\checkmark$ Ignorar los deseos y opiniones de las mujeres y sus familiares.

$\checkmark$ No prestar la atención médica adecuada y a tiempo.

$\checkmark$ Presionar para que decidan uno u otro método de anticoncepción.

$\checkmark$ Esterilizaciones forzadas.

\section{La violencia obstétrica}


La violencia contra la mujer, explica Fernandez (2017), constituye e implica una violación a los derechos humanos. La cual se puede definir como el maltrato que sufre la mujer embarazada al ser juzgada, atemorizada, vejada, lastimada física y emocionalmente, también se incluye el hecho de no ser respetada en sus decisiones. Es recurrente en los hospitales, y se da en todas las esferas de la sociedad.

Las mujeres en el embarazo y la procreación se encuentran en un estado vulnerable; por lo tanto la Convención sobre la eliminación de todas las formas de discriminación en contra de la mujer (CEDAW, 2013), pide a los estados adoptar medidas que eliminen la discriminación en contra la mujer y recomienda que los servicios de atención medica garanticen el libre acceso a servicios relacionados durante el embarazo, parto y puerperio, además de contar con servicios de maternidad gratuitos y sin riesgo, los cuales también deben de ser libres de discriminación racial y edad.

La violencia obstétrica se presenta sin importar religión, edad, nivel socio-económico y educativo; esto quiere decir que todas las mujeres son vulnerables. El estado tiene la obligación de proveer servicios de salud materna desde la perspectiva de derechos humanos y en este aspecto la Comisión Interamericana de Derechos Humanos, ha recomendado garantizar el acceso a la atención profesional durante el parto y el periodo posterior, incluyendo servicios de obstetricia de emergencia, especialmente a los grupos de mujeres en condiciones de exclusión, respetando las necesidades específicas y decisiones, al respecto me refiero a los usos y costumbres de cada región.

El personal médico, apunta Magnone (2011), debe estar capacitado en todos sentidos, tener un trato de calidad y humanizante, ya que este tipo de violencia se podría considerar una forma de tortura, así como la esterilización forzada sin consentimiento de las mujeres y la negativa de poseer servicios de salud sexual y reproductiva. También se considera violencia obstétrica alterar el proceso natural del parto de bajo riesgo mediante el uso de técnicas que aceleren el nacimiento sin el consentimiento de la madre (ya sea de manera verbal o escrita), al igual que se realice el parto vía cesárea existiendo condiciones para el parto natural y la inadecuada atención a emergencias obstétricas.

\section{Deshumanización del parto}


El término "humanización del parto" no se limita a la posición vertical o a la modalidad del parto en agua. Se trata de una visión holística del proceso de gestación que involucra el embarazo, el parto y el nacimiento, donde se respeta la fisiología de dicho proceso. Dentro del parto humanizado se presentan alternativas como el parto vertical, en el cual la mujer puede estar de pie, de rodillas o agachada, en la cama, el piso o una tina con agua. Lo importante es que la mujer tenga la libertad de decidir cómo quiere traer al mundo a su hijo. Conocer y elegir entre las opciones disponibles, en qué lugar (su casa o una clínica), acompañada por qué personas (pareja, hijos, madre, amigos, etc.), bajo qué ambiente y en qué condiciones.

Ecuador, afirma Johannsen (2016), tiene una tasa promedio de $41 \%$ de cesáreas que, representa un incremento de aproximadamente el $60 \%$ en un promedio de 8 años. Otros países como Brasil y México tienen tasas aún más altas de cesáreas, de hasta el 80\%. En este entorno, una atención participativa, humana y respetuosa del parto requiere un esfuerzo adicional por parte de las embarazadas en términos de información, tiempo, costo y valor. De todas formas, las embarazadas tienen menos opciones reales en el sector público que en el privado de tener un parto humanizado. Las pacientes del sector privado pueden escoger su obstetra de confianza que atiende de la manera que prefieren y quien está presente en el momento de dar a luz; mientras que, en el sector público, la dinámica de la atención es menos personalizada y no se cuenta con la posibilidad de la libre elección.

\section{Metodología}

La metodología fue de tipo descriptiva de campo y con un diseño no experimental. La población la constituyeron 150 pacientes que solicitaron los servicios de los hospitales Matilde hidalgo de Procel, Mariana de Jesús y General Guasmo Sur, durante el periodo de agosto 2017 hasta abril 2018. Los datos se obtuvieron posterior a la aplicación de un instrumento tipo cuestionario a través de la técnica de la encuesta en el área de la consulta externa, sala de post parto y hospitalización. El análisis de los datos se hizo considerando la estadística descriptiva de las frecuencias absolutas y relativas.

\section{Análisis y discusión de los resultados}


Posterior al procesamiento estadístico de los datos, los resultados se presentan en tablas con sus respectivos análisis.

Tabla 1. Grado de violencia obstétrica en Hospitales Matilde Hidalgo de Procel, Mariana de Jesús y General Guasmo Sur (agosto 2017 - abril 2018)

\begin{tabular}{|c|c|c|c|c|c|c|c|c|}
\hline \multirow{2}{*}{ Hospitales y centros } & \multicolumn{2}{|c|}{ Alta } & \multicolumn{2}{c|}{ Media } & \multicolumn{2}{c|}{ Baja } & \multicolumn{2}{c|}{$\begin{array}{c}\text { Total usuarias } \\
\text { encuestas en } \\
\text { hospitales }\end{array}$} \\
\cline { 2 - 9 } & FR & $\%$ & FR & $\%$ & FR & $\%$ & FR & $\%$ \\
\hline $\begin{array}{c}\text { Matilde Hidalgo de } \\
\text { Procel }\end{array}$ & 94 & $62,67 \%$ & 3 & $2 \%$ & 3 & $2 \%$ & 100 & $66,67 \%$ \\
\hline $\begin{array}{c}\text { Mariana de Jesús } \\
\text { General Guasmo Sur }\end{array}$ & 15 & $10 \%$ & 3 & $2 \%$ & 12 & $8 \%$ & 30 & $20 \%$ \\
\hline Total & $8,66 \%$ & 1 & $0,67 \%$ & 6 & $4 \%$ & 20 & $13,33 \%$ \\
\hline
\end{tabular}

Fuente: Elaboración propia

En la tabla 1, se presenta el análisis descriptivo del grado de violencia obstétrica en los hospitales Matilde Hidalgo de Procel, Mariana de Jesús y General Guasmo Sur, en el lapso de agosto 2017 - abril 2018, en la misma se indica que existe una alta percepción de violencia obstétrica en el hospital Matilde Hidalgo de Procel reflejado en un 62.67\%, cifra que es altamente significativa respecto a la de otros hospitales de la ciudad de Guayaquil.

Tabla 2. Conducta de los profesionales durante la atención del parto en hospitales Matilde Hidalgo de Procel, Mariana de Jesús y General Guasmo Sur (agosto 2017 - abril 2018)

\begin{tabular}{|c|c|c|c|c|c|c|}
\hline \multirow{2}{*}{ Conductas de profesionales } & \multicolumn{2}{|c|}{ Si } & \multicolumn{2}{c|}{ No } & \multicolumn{2}{c|}{ Total } \\
\cline { 2 - 7 } & FR & $\%$ & FR & $\%$ & FR & $\%$ \\
\hline Comentarios inapropiados & 89 & $59,33 \%$ & 61 & $40,67 \%$ & 150 & $100 \%$ \\
\hline Uso de sobrenombres & 116 & $77,33 \%$ & 34 & $22,67 \%$ & 150 & $100 \%$ \\
\hline Insultos & 54 & $36 \%$ & 96 & $64,00 \%$ & 150 & $100 \%$ \\
\hline
\end{tabular}


En la tabla 2, se presenta el análisis descriptivo de la conducta de los profesionales durante la atención del parto en los hospitales Matilde Hidalgo de Procel, Mariana de Jesús y General Guasmo Sur, en el lapso de agosto 2017 - abril 2018, en la misma se observa comentarios inapropiados en el parto, que vulneraron su integridad, cuando se consultó sobre el uso de sobrenombres el $77.33 \%$ respondió afirmativamente, en lo que respecta el uso de insultos 54 usuarias que representan el 36\% respondieron que fueron agredidas verbalmente. También se observa entre sus resultados que el $59.33 \%$ de usuarias fueron víctimas de comentarios desagradables durante el proceso del trabajo de parto y parto. Datos similares han sido reportados por otros autores, previamente sobre el comportamiento del profesional de la salud durante la atención obstétrica. (Figueroa Palomino, y colaboradores, 2017).

Al comparar con otros estudios, un $37.7 \%$ de 45 usuarias encuestadas presentaron algún tipo de temor al limitar sus preguntas sobre lo que está pasando o el procedimiento que se le vaya a realizar, debido a que pueden encontrar como respuesta comentarios desagradables. Por otro lado, Pereira, (2015), en su estudio con 1755 usuarias encuestadas, se evidenció que el 14.1\% respondieron afirmativamente al preguntarle si habían sido víctimas de comentarios inapropiados, violentando la parte psicológica de las usuarias. Nuestros resultados presentan un porcentaje elevado de conducta profesional inadecuada, posiblemente debido a que la sobre carga laboral incrementa el estrés laboral conllevando que los profesionales utilicen comentarios inadecuados.

Tabla 3. Atención humanizada del embarazo, parto, post-parto hospitales Matilde Hidalgo de Procel, Mariana de Jesús, y General Guasmo Sur (agosto 2017 - abril 2018)

\begin{tabular}{|c|c|c|c|c|c|c|}
\hline \multirow{2}{*}{ Atención humanizada } & \multicolumn{2}{|c|}{ SI } & \multicolumn{2}{c|}{ NO } & \multicolumn{2}{c|}{ TOTAL } \\
\cline { 2 - 7 } & FR & $\%$ & FR & $\%$ & FR & $\%$ \\
\hline Posición de preferencia & 42 & $28,00 \%$ & 108 & $72,00 \%$ & 150 & $100 \%$ \\
\hline Acompañamiento & 41 & $27,33 \%$ & 09 & $72,67 \%$ & 150 & $100 \%$ \\
\hline Apego precoz & 52 & $34,67 \%$ & 98 & $65,33 \%$ & 150 & $100 \%$ \\
\hline
\end{tabular}


En la tabla 3, se presenta el análisis descriptivo de la atención humanizada del embarazo, parto y postparto durante la atención del parto en los hospitales Matilde hidalgo de Procel, Mariana de Jesús y General Guasmo Sur, en el lapso de agosto 2017 - abril 2018, en la misma se puede apreciar que 108 usuarias, que representan el 72\%, indicaron que no pudieron optar por la posición de preferencia en el momento del parto, violentando el parto en libre posición; cuando consultamos sobre si estuvo acompañada por un familiar de confianza 109 usuarias que representan el 72.67\% respondió que se les negó el acompañamiento, y en lo que respecta al apego precoz, 98 usuarias, que representan el $65.33 \%$, respondieron que no se les dio la oportunidad de éste, violentando psicológicamente a la usuaria al negarle una atención humanizada en su trabajo de parto.

En lo referente al acompañamiento durante el trabajo de parto, se pudo evidenciar que en el $72.67 \%$ de los casos no dio cumplimiento a este. Cantero y col, (2016), mostró que al 46.5\% de las usuarias se les negó el estar acompañada en el momento del trabajo de parto o parto, incumpliendo el acompañamiento; mientras que Ibáñez (2016), obtuvo que un 63\% de las usuarias que fueron encuestadas, pusieron en manifiesto que no se cumplió el acompañamiento, alegando los profesionales de la salud que el acompañante interrumpe en el momento de la praxis medica. Figueroa y col (2017), determinaron que existió un alto porcentaje de violación al proceso de acompañamiento durante el trabajo de parto. Los resultados muestran que existe por parte de los profesionales de salud poca disposición para que se dé un acompañante durante el trabajo de parto, alegando que no es permitido el acompañamiento por políticas de las instituciones públicas.

En lo que respecta a la libre posición para el parto, el 72\% de usuarias no tuvieron la opción de escoger la posición deseada. En el trabajo de Figueroa Palomino y col (2017), se encontró que al $52 \%$ de las usuarias encuestadas no se les admitió elegir la posición de preferencia, no permitiéndoles a las usuarias optar por una posición de su elección. Soto y col (2016), reportó que el $27.1 \%$ de los datos son inferiores, pero importantes a la vez debido a que se vulnera optar una libre posición para el parto. Los resultados son extremadamente altos, dado que al evaluar el apego precoz encontramos que $65.33 \%$ de las puérperas no se les brindo esta opción, datos que superan a los reportados por Figueroa y col (2017), en el cual solo al 26.7\% no se les dio la oportunidad de tener el contacto inmediatamente con el recién nacido. Pereira, (2015), hace 
referencia sobre el apego precoz, donde se obtuvo que al 3.6\% de las usuarias se les negó este derecho, aunque el porcentaje es mínimo no deja de ser importante debido a que todas las usuarias deben de tener el apego precoz inmediatamente a la salida de su hijo (a), salvo que exista alguna complicación neonatal para que no pueda ser aplicado el apego precoz inmediatamente. En base a los resultados, se evidencia que se violenta la parte afectiva y psicológica de las usuarias al momento de ya saber que nació su hijo (a), debido a porcentajes preocupantes de poca sensibilidad por parte de los profesionales de salud, el profesional de la salud ha creado un "estereotipo" de como las usuarias deben de parir, evitando que se adopten otras posturas en las cuales algunos profesionales no presentan experticia.

Tabla 4. Aplicación de procedimientos para acelerar el trabajo de parto hospitales Matilde hidalgo de Procel, Mariana de Jesús, y General Guasmo Sur (agosto 2017 - abril 2018)

\begin{tabular}{|c|c|c|}
\hline Tipo de procedimiento & FR & $\%$ \\
\hline Rotura artificial de membranas & 17 & $11,33 \%$ \\
\hline $\begin{array}{l}\text { Administración de medicamentos para } \\
\text { acelerar el trabajo de parto }\end{array}$ & 49 & $32,67 \%$ \\
\hline Compresión de abdomen durante el pujo & 12 & $8,00 \%$ \\
\hline Episiotomía & 72 & $48,00 \%$ \\
\hline Total & 150 & $100 \%$ \\
\hline
\end{tabular}

En la tabla 4, se presenta el análisis descriptivo de la aplicación de procedimientos para acelerar el trabajo de parto durante la atención del parto en los hospitales Matilde Hidalgo de Procel, Mariana de Jesús y General Guasmo Sur, en el lapso de agosto 2017 - abril 2018, en la misma se puede apreciar que de las 150 usuarias, a 72 que representan el 48\% se les realizó la episiotomía para acelerar el expulsivo. Al preguntar sobre la administración de medicamentos para acelerar el trabajo de parto 49 usuarias que representar el $32.67 \%$ respondieron afirmativamente, en lo que respecta a ruptura artificial de membranas 17 usuarias que representan el $11.33 \%$ respondieron que realizaron dicho procedimiento sin consentimiento de la usuaria, y 12 usuarias que representan el $8 \%$ manifestaron que se les comprimió el abdomen de una manera brusca violentando su integridad física. 
En relación a la aplicación de procedimientos para acelerar el trabajo de parto, la rotura artificial de membranas obtuvo el 11.33\%, al revisar al revisar el trabajo de Terán, Castellanos, González Blanco y Ramos (2013), realizado en la ciudad de Caracas Venezuela, a las usuarias encuestadas se les realizó este procedimiento, obteniendo un 19.5\%, violentando que el parto sea lo más fisiológico posible. Soto y col (2016), obtiene que el $76.4 \%$ de uso de rotura artificial de membranas como procedimiento rutinario para acelerar el trabajo de parto, a sabiendas que el parto es un proceso fisiológico que debe de evolucionar espontáneamente. Nuestros resultados muestran un bajo empleo de procedimiento medico artificial lo cual indica que se promueve parto humanizado.

En el reporte de procedimientos médicos para acelerar el trabajo de parto, tenemos que al $32.67 \%$ se les administró conducción mediante oxitócicos, sin indicarles por qué eran necesarios aplicarlos. Camacaro, Ramírez, Lanz y Herrera (2014), reportaron que al 83.3\% de las usuarias se les aplicó oxitócicos para acelerar el trabajo de parto y el parto, observando que existió un alto índice de uso medicamentoso para acelerar este proceso fisiológico, como lo es el parto. Los resultados muestran similitud con los presentados por Terán y col (2013), en los cuales el uso de la medicalización del parto se mantiene en porcentajes considerables.

Al hacer referencia sobre el uso de la maniobra de Kristeller, se indicó que se aplicó al 8\%, cabe señalar que Terán, Castellanos, González Blanco y Ramos (2013), establecieron que al 15.8\% de las usuarias, se les realizó dicha maniobra, poniendo en riesgo la parte física de las usuarias al soportar este tipo de procedimiento. García y Peña (2015), manifestaron que $12 \%$ de las usuarias sufrieron un trauma físico debido a la aplicación de esta maniobra, dato que es menor a la de nuestros resultados. Becerra y Chauca (2015), afirmaron que al $77.6 \%$ de las usuarias se les aplicó esta maniobra, inclusive siendo en reiteradas ocasiones. Nuestros resultados son menores a los reportados por otros autores previamente descritos, sin embargo, es un procedimiento médico, el cual está contraindicado en la Ley Orgánica de la Atención Humanizada del Embarazo, parto y post parto.

En lo que respecta a la práctica de la episiotomía, tenemos que al $48 \%$ de las usuarias que participaron en la investigación se les realizó este procedimiento sin informarles, datos que superan a los obtenidos por Terán y col. (2013), solo al 20\% de las participantes se les realizó 
este proceso haciéndolo selectivo, mientras que es menor a los reportados por Camacaro, Ramírez, Lanza y Herrera (2014), quienes obtuvieron que al 75\% se le realizó este procedimiento siendo esta una cifra alarmante a las antes señaladas, dado que se pone en riesgo la integridad fisica de las usuarias.

\section{Conclusiones}

Posterior al análisis y discusión de los resultados y en atención a los objetivos del estudio, se generaron las siguientes conclusiones:

- La conducta de los profesionales durante la atención del parto no es la adecuada, ya que se pudo comprobar la existencia de violencia obstétrica, al utilizar términos inadecuados, agresiones verbales y realizar procedimientos innecesarios sin indicarles para qué y por qué de la aplicación de éstos.

- Se pudo evidenciar que la Ley Orgánica para la atención humanizada del embarazo, parto y post parto está escrita, pero no se cumple en su totalidad, lo cual indica que se están violando los derechos de las usuarias, al no permitirles el acompañamiento durante el parto, no elegir la posición deseada, y no darles la oportunidad del apego precoz, eventos que en lo posterior podrían generar un trauma psicológico en nuestras mujeres, y que puede ser objeto de otra investigación.

- El uso de Misoprostol y Oxitocina para acelerar el trabajo de parto fue alto, lo que nos indica que, en nuestras instituciones, todavía se efectúa la medicalización de parto, sin permitir que este fluya de manera natural.

- Se constató la poca capacitación, en los profesionales, sobre la atención del parto que refleje el profesionalismo y humanismo que debe caracterizar al profesional que atiende a la mujer gestante, a la parturienta y a la puérpera.

- Existe desconocimiento acerca de lo contemplado por la Ley Orgánica para la atención humanizada del embarazo, parto, post-parto, para otorgar a la usuaria la mejor atención obstétrica, donde no se le vulneren sus derechos. 


\section{Referencias bibliográficas}

Becerra, N \& Chauca, Y. (2015). Maniobra Kristeller, sus consecuencias en la madre y condiciones bioéticos en el Hospital Hipólito Unanue - El Agustino durante el periodo agosto 2015. REPOSITORIO ACADEMICO USMP, 70.

Camacaro, M., Ramírez, M., Lanza, L., \& Herrera, M. (2014). Conductas de rutina en la atención al parto constitutivasde violencia obstétrica. Red de Revistas Científicas de América Latina y el Caribe, España y Portuga, 9.

Cantero, A., Fiuri, L., Furfaro, K., Jankovic, P., Llompart, V., \& San Martín, E. (2016). ACOMPAñAMIENTO EN SALA DE PARTOS: REGLA O EXCEPCIóN. Sistema de Información CientíficaRed de Revistas Científicas de América Latina y el Caribe, España y Portugal, 6.

Pereira, C. (2015). Violencia obstétrica desde la perspectiva de la paciente. Revista de Obstetricia y Ginecología de Venezuela, 5-8.

El Senado y Cámara de Diputados de la Nación Argentina. (11 de 03 de 2009). LEY DE PROTECCION INTEGRAL A LAS MUJERES. Obtenido de LEY DE PROTECCION INTEGRAL A LAS MUJERES:

https://www.oas.org/dil/esp/Ley_de_Proteccion_Integral_de_Mujeres_Argentina.pdf

Fernandez, J. (2017). Violencia obstétrica en contextos de crisis de salud. Amnistia Internacional, 6.

Figueroa, O., Hurtado, R., Valenzuela, D., Bernal J., Duarte, C., \& Cazares, F. (2017). Violencia Obstétrica: percepción de las usuarias sonorenses. Sanus, 8.

Garcia, H., \& Peña , A. (2015). Incidencia de lesiones asociadas. Revista Médica del IMSS, 7.

Ibáñez Díaz. (2016). Opinión de las gestantes, sus acompañantes y los profesionales de salud que les atienden sobre el acompañamiento en el parto. Dialnet, 7.

Magnone Alemán, N. (2011). Derechos sexuales y reproductivos en tensión: intervencionismo y violencia obstétrica. Departamento de Trabajo Social-FCS, 24. 
Soto, C., Teuber, H., Cabrera, C., Marín, M., Cabrera, J., \& Araneda, H. (2016). EDUCACIÓN PRENATAL Y SU RELACIÓN CON EL TIPO DE PARTO: UNA VÍA HACIA EL PARTO NATURAL. Rev. Child. Obstet. Ginecol. v.71 n.2 Santiago 2016, 6.

Teran, P., Castellanos, C., Gonzalez Blanco, M., \& Ramos, D. (2013). Violencia obstétrica: percepción de las usuarias. Rev Obstet Ginecol Venez vol.73 no.3 Caracas set. 2013, 10.

CEDAW (2013). Convención sobre la eliminación de todas las formas de discriminación en contra de la mujer https://www.google.com.ec/search?q=cedaw+que+es\&oq=CEDAW\&aqs=chrome.2.69i57j015.40 92j0j7\&sourceid=chrome\&ie=UTF-8. Recuperado en febrero 2017.

OMS. (2014). Organización Mundial de la https://www.google.com.ec/search?ei=mocW5S8HsOW5gL8q7GwCA\&q=Organización++Mun dial++de++la++Salud+y+el+parto.++\&oq=Organización++Mundial+Recuperado en julio 2017.

Johannsen, J. (2016). Parto humanizado, la información es poder. https://blogs.iadb.org/salud/2016/08/30/parto-humanizado/. Recuperado en marzo 2017.

LOAPH. (2016). Ley orgánica para la atención del Parto Humanizado del Ecuador. https://www.google.com.ec/search?q=ley+orgánica+para+la+atención+del+Parto+Humanizado+ del+Ecuador\&oq=ley+orgánica+para+la+atención+del+Parto+Hum. Recuperado en octubre 2017. 